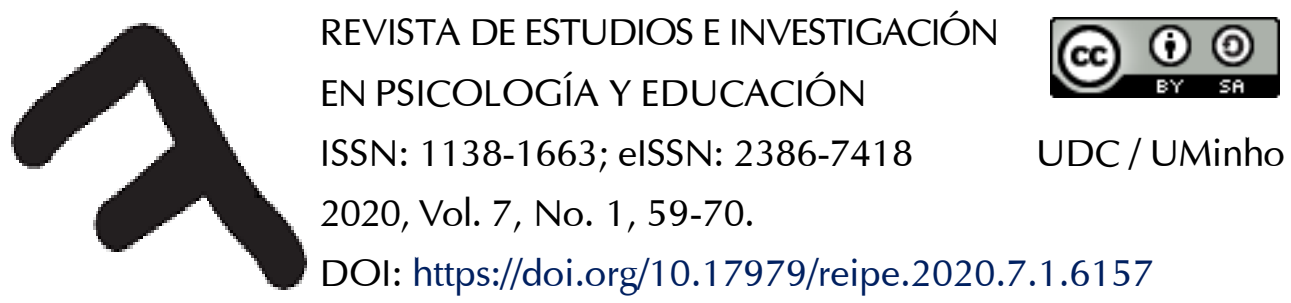

\title{
Estructura de un cuestionario para evaluar la ansiedad hacia las matemáticas en estudiantes de ingeniería
}

\author{
Structure of a questionnaire to assess anxiety towards mathematics \\ among engineering students
}

\author{
Carlos Soneira (D), Dorinda Mato-Vázquez \\ Universidade da Coruña
}

\begin{abstract}
Resumen
En este trabajo se pretendía determinar la estructura factorial de un cuestionario específico para evaluar la ansiedad hacia las Matemáticas en estudiantes universitarios de Ingeniería, así como estudiar los resultados de su implementación. Está motivado por el hecho de que, siendo el dominio de las Matemáticas esencial en las Ingenierías, en los últimos años la ansiedad está siendo considerada la raíz de muchos casos de aversión y fracaso académico en la disciplina. Para ello, en una primera fase se ha realizado un análisis factorial exploratorio con 627 estudiantes de Educación Secundaria para validar un cuestionario en el que se aplica/utiliza una escala tipo Likert. En una segunda fase, se ha aplicado un análisis factorial confirmatorio a una muestra de 215 estudiantes de Ingeniería. Como resultado se han obtenido dos factores: "Ansiedad ante la evaluación", y "Ansiedad hacia los números y operaciones matemáticas". Los resultados reflejan una relación entre ambos factores y mayor "Ansiedad ante la evaluación". Esta relación entre factores y los valores alcanzados en ciertos ítems sugieren que la evaluación continua puede eliminar parte de la distorsión que introduce la ansiedad al valorar la competencia matemática.
\end{abstract}

Palabras clave: ansiedad; educación universitaria; evaluación; matemáticas

\begin{abstract}
The aim of this article is to establish the factor structure of a questionnaire for the assessment of anxiety towards mathematics among undergraduate engineering students, and to study the results of its implementation. Even though mathematics is essential in engineering, anxiety is increasingly seen as one of the main causes of aversion to the subject and high failure rates. The first phase of the study consisted of an exploratory factor analysis involving 627 secondary school students, in order to validate a Likert-type scale questionnaire. The second phase consisted of a confirmatory factor analysis with a sample involving 215 engineering students, from which two factors were obtained: 'anxiety towards assessment' and 'anxiety towards numbers and mathematical operations'. The results reveal a relationship between the two factors and higher levels of 'anxiety towards assessment'. This relationship and other values obtained from the questionnaires suggest that continuous assessment may help to reduce the distorting effect of anxiety on mathematical performance.
\end{abstract}

Keywords: anxiety; university; assessment; mathematics

Carlos Soneira (Dhttps://orcid.org/0000-0002-2902-268X y Dorinda Mato (Dhttps://orcid.org/0000-0002-8309-2400: Departamento de Pedagoxía e Didáctica, Facultade de Ciencias da Educación, Universidade da Coruña. Campus de Elviña s/n, 15071 A Coruña, España. 
Siendo muchas las alteraciones que cabría englobar bajo el epígrafe de ansiedad juvenil, este trabajo se centra en la que, de forma genérica, se puede denominar ansiedad asociada a la vida académica en la escuela, y que influye negativamente en el rendimiento de los individuos. En efecto, como señala Kazelskis (2000), el alumnado ansioso está excesivamente preocupado por su progreso en clase y lo que el profesorado espera de él. Este fenómeno constituye en el proceso de enseñanza-aprendizaje de las Matemáticas un importante factor de distorsión al provocar sentimientos subjetivos de tensión, aprehensión, preocupación y sobre-activación del sistema nervioso.

La Matemática es, en la actualidad, una asignatura básica en los planes de estudio de todos los niveles educativos y países, pues se hace necesaria para entender e interpretar el mundo (Álvarez y Ruíz, 2010). Sin embargo, si bien constituyen una herramienta tanto en otras disciplinas científicas como en múltiples actividades profesionales, es la materia que causa más fracasos escolares en cualquier etapa educativa, de acuerdo a lo manifestado por la National Council of Teachers of Mathematics (NCTM, 2003). En efecto, numerosos estudios han señalado que muchos/as estudiantes experimentan crisis de pánico, miedo, angustia y sudores cuando intentan hacer algo relacionado con las Matemáticas. Algunos/as se sienten enfermos/as y desesperados/as, como reacción a no entender lo que se les pide, dejar algo sin terminar o ser humillados/as públicamente (Anderson y Schunn, 2000; Iossi, 2007; Yara, 2009). Por consiguiente, los beneficios que resultan de cambiar la ansiedad por confianza en Matemáticas no son sólo profesionales y económicos, sino también relativos al bienestar emocional.

Aunque hay poco acuerdo en la conceptualización del constructo Ansiedad hacia las Matemáticas, la aversión hacia la asignatura y los efectos emocionales más intensos que surgen en algunas personas al tener que relacionarse con las Matemáticas, hace que se acepte universalmente su importancia.

Richardson y Suinn (1972) la describen como sentimientos de tensión y ansiedad que interfieren con la manipulación de números y la solución de problemas matemáticos en la vida cotidiana y académica. Por su parte, Carmona (2004) la define como una fobia específica, esto es, un miedo desmesurado hacia las Matemáticas que puede manifestarse a través de una preocupación excesiva, pensamientos perturbadores y alteración fisiológica en determinadas situaciones escolares. La ansiedad hacia las Matemáticas es el resultado de diferentes factores; por ello las causas son difíciles de identificar (Akey, 2006; Auzmendi, 1992; Fennema y Sherman, 1976). Puede ser la falta de confianza en la competencia matemática propia (Birgin, Catlioglu, Costu y Aydin, 2009) o debida a factores sociales, educativos o ambientales (Delgado Espinoza y Fonseca, 2017; Swars, Daane y Giesen, 2010). Por ejemplo, experiencias negativas en el aula, presión familiar, metodologías de enseñanza, o la cercanía para realizar un examen (Yara, 2009).

Para explicar el funcionamiento de la ansiedad y cómo ésta obstruye posteriores aprendizajes en Matemáticas, Tobías (1985) concibe el cerebro humano como una estructura con tres partes: el Input, el Banco de Memoria y una Vía de Enlace entre las anteriores. Si el sistema funciona bien, ante un nuevo problema de Matemáticas se rescata de la memoria el conocimiento adecuado y se recorre el camino de comprensión para llegar a la solución.

Así, puede que la memoria y el camino de comprensión funcionen adecuadamente pero que, cada vez que aparece un nuevo problema, se produzca una reacción emocional del tipo: "nunca seré capaz de resolver este problema". Esto bloquea el camino de la comprensión e imposibilita recordar, con la consiguiente pérdida de auto-confianza en su inteligencia. No obstante, el conflicto no radica en la falta de capacidad a nivel intelectual sino en el bloqueo emocional.

Altos niveles de ansiedad disminuyen la atención, concentración, retención y la eficiencia en el aprendizaje, bajando el rendimiento escolar (Van Eerde, 2003; Immordino-Yang y Damasio, 2007). De hecho, la mayoría de estudios asocian un bajo nivel de rendimiento al alto nivel de ansiedad, (Swars et al., 2010), ya que el nerviosismo del alumnado provoca dificultades tanto para aprender como para expresar lo aprendido (Hancock, 2001), aumentando la ansiedad a medida que se acerca el examen si este se considera difícil (Clark y Beck, 2012).

Otro fenómeno observado es que la ansiedad hacia las Matemática provoca que se evite matricularse en itinerarios de Bachillerato o grados universitarios con asignaturas de Matemáticas (Anderson y Schunn, 2000). 
Ashcraft (2002) analiza la ansiedad hacia las Matemáticas entre estudiantes universitarios pertenecientes a diferentes áreas de conocimiento (Ciencias Físicas, Sociales y Humanas), obteniendo niveles más altos en el estudiantado menos relacionado con el área de Matemáticas. Es más, muchos estudios reportan resultados análogos, como los de Hancokc (2001) o los de Pérez-Tyteca, Castro, Rico y Castro (2011).

Cabe tener también en cuenta que el efecto negativo es proporcional al nivel de ansiedad, especialmente en las tareas complejas, cuando los límites de tiempo son ajustados, tal como indican Nortes y Nortes (2014). Esto tendría una especial trascendencia en la actividad profesional asociada a algunos grados, como la de los de Ingeniería, donde los tiempos de entrega del trabajo muy ajustados son habituales. Pero las ingenierías son cada vez más relevantes, tanto en las sociedades con alto desarrollo tecnológico como en las que aspiran a serlo, y si bien las Matemáticas son imprescindibles en los grados de ingeniería, diversos estudios reportan elevados niveles de suspensos y ansiedad hacia las Matemáticas entre el alumnado (Swars et al., 2010). Esto hace que su investigación específica despierte interés internacionalmente (Álvarez y Ruíz, 2010; EcciusWellmann y Lara-Barragán, 2016).

El presente trabajo tenía como objetivo validar un cuestionario específico para evaluar la ansiedad hacia las Matemáticas en los/las estudiantes de grados de Ingeniería. Por una parte, el conocimiento de la estructura del constructo permite una mayor adaptación de la praxis docente y una mejor interpretación de los resultados de evaluación. En este sentido, dado que tal conocimiento será posible en la medida en que se disponga del instrumento adecuado, se necesita un instrumento específico para la población objeto de estudio. Por otra parte, se pretendía aportar un instrumento que puedan usar los/las docentes universitarios/as para valorar los niveles de ansiedad matemática de sus estudiantes de Ingeniería.

\section{Método}

El estudio realizado puede clasificarse como descriptivo y enmarcado dentro de una investigación de tipo transeccional según la clasificación de Dankhe (1989). Se ha seguido un diseño secuencial de dos fases, con un Análisis Factorial Exploratorio (AFE) seguido de un Análisis Factorial Confirmatorio (AFC).

\section{Participantes}

En la primera fase participaron de forma voluntaria 627 estudiantes de los dos últimos cursos de la Educación Secundaria Obligatoria (ESO) de ambos sexos entre los 14 y los 16 años de edad $(M=15.59 ; D T$ $=0.86$ ), tanto de centros públicos como privados, de una ciudad de tamaño medio del Estado Español. Son los dos últimos cursos académicos en los que todo el alumnado tiene clase de Matemáticas.

En la segunda fase participaron, 215 estudiantes de primer curso de un Grado en Ingeniería (GI) de la universidad pública situada en la misma ciudad, mediante un sistema de muestreo de conveniencia. El $78.5 \%$ tenían entre 18 y 22 años, el $17.1 \%$ entre 23 y 27 y el resto edades superiores $(M=21.29 ; D T=4.01)$. En cuanto a la distribución por sexos, el $17.74 \%$ eran mujeres y el $82.26 \%$ hombres. En la población origen, según los datos del curso académico en el que se realizó el estudio publicados por la universidad y correspondientes a la titulación, entre los estudiantes de nuevo ingreso el $14.17 \%$ eran mujeres y el $85.83 \%$ hombres.

El primer curso del Grado incluía como obligatorias, en el primer cuatrimestre, las materias de Cálculo y Matemática Discreta, y en el segundo curso Álgebra y Estadística. Por lo tanto, los/las estudiantes que eligieron esta carrera eran conocedores/as de la conveniencia de una formación matemática sólida. De hecho, de entre los participantes, 171 provenían del Bachillerato Tecnológico, cuya orientación incluye la formación matemática más amplia, y los 44 restantes de otros itinerarios de Bachillerato u otras formas de acceso a la universidad.

\section{Instrumentos}

El instrumento utilizado para la primera fase (alumnado de $3^{\circ}$ y $4^{\circ}$ de ESO) fue el cuestionario diseñado por Muñoz-Cantero y Mato-Vázquez, (2006). Estaba formado por 24 ítems con respuesta tipo Likert de 1 a 5 , 
agrupados en cinco factores: "Ansiedad ante la evaluación", "Ansiedad ante la temporalidad", "Ansiedad hacia la comprensión de problemas matemáticos", "Ansiedad hacia los números y las operaciones Matemáticas" y "Ansiedad hacia situaciones Matemáticas de la vida real".

A partir de los resultados del Análisis Factorial Exploratorio del presente trabajo, se eliminaron 5 ítems de la escala original, pasando de 24 a 19, distribuidos en tres factores: "Ansiedad ante la evaluación", "Ansiedad hacia la temporalidad" y "Ansiedad hacia los números y las operaciones Matemáticas". Este cuestionario modificado fue el utilizado en la segunda fase para el Análisis Factorial Confirmatorio con estudiantes de GI.

No se aplicó directamente el cuestionario original (Muñoz-Cantero y Mato-Vázquez, 2006) porque este había sido diseñado y validado con estudiantes con edades a partir de 12 años, por lo que existían diferencias en el nivel de maduración personal entre ambas poblaciones.

\section{Procedimiento}

Los datos fueron recogidos de forma presencial en las aulas delante del profesorado de forma anónima y voluntaria. Previamente se explicó al alumnado los objetivos de la investigación y las instrucciones necesarias.

En la primera fase se realizó un Análisis Factorial Exploratorio (AFE) con estudiantes de los dos últimos cursos de la ESO mediante la aplicación de la escala de Muñoz-Cantero y Mato-Vázquez (2006) elaborada a partir del MARS (The Mathematics Anxiety Rating Scale) de Richardson y Suinn (1972).

En la segunda fase, se utilizaron los resultados de la primera para realizar un Análisis Factorial Confirmatorio (AFC) con estudiantes de GI. Además de contrastarse el modelo propuesto, fueron realizadas diversas modificaciones del mismo para mejorar el ajuste y se compararon modelos alternativos. Finalmente, se calcularon los estadísticos descriptivos y se abordó la correlación entre factores.

\section{Análisis de datos}

En la realización del AFE, siguiendo las indicaciones de Blunch (2008), se usó la extracción por ejes principales con rotación ortogonal varimax. Para retener factores, se tomó como primer criterio el de los autovalores $>1$, y se recalcularon todos los índices y coeficientes después de cada cambio.

En la realización del AFC en la segunda fase de la investigación, se empleó el método de unweighted least squares. Para estudiar el ajuste del modelo y comparar distintos modelos alternativos, se usaron diversos índices. En concreto, $\chi^{2}=(\mathrm{n}-1)[0.5 \operatorname{Tr}((S-\Sigma) 2)]$, siendo $\operatorname{Tr}$ la función traza, $S$ la matriz de covarianzas observadas y $\Sigma$ la matriz de covarianzas reproducidas por el modelo (nótese que la fórmula para $\chi^{2}$ depende del método de estimación de los parámetros, en nuestro caso el ULS); el Índice residual de la raíz cuadrada media (RMR); el Índice de bondad de ajuste (GFI); el Índice de bondad de ajuste ponderado (AGFI); el Índice de ajuste normalizado (NFI); el Índice de ajuste relativo (RFI); la razón de parsimonia (PRATIO); el Índice de ajuste parsimónico (PGFI), y el Índice de ajuste parsimónico normalizado (PNFI).

Puesto que en la realización del AFC la pertinencia de los distintos procedimientos a seguir dependía de los sucesivos resultados intermedios obtenidos, remitimos al lector a la sección de Resultados para consultar los detalles del proceso. La correlación entre factores fue estudiada mediante el coeficiente de correlación de Pearson.

\section{Resultados}

\section{Análisis Factorial Exploratorio}

A partir del cuestionario de Muñoz-Cantero y Mato-Vázquez (2006) se eliminaron sucesivamente varios ítems por su baja comunalidad. En concreto "Me siento nervioso cuando me dan las preguntas del examen de Matemáticas" y "Me siento nervioso cuando reviso el ticket de compra de después de haber pagado", con comunalidades .47 y .38 respectivamente.

Tras este proceso, se obtuvieron 4 factores que explicaban el $80.84 \%$ de la varianza. Sin embargo, los tres ítems que podrían corresponder al cuarto factor, tenían cargas más altas o prácticamente iguales en otros factores. Esto dificultaría la interpretación del modelo en base a la teoría, por lo que se eliminaron. 
Finalmente, con 19 ítems, se ha obtenido que todas las comunalidades son mayores que .67, nivel considerado alto, y la escala es fiable con un coeficiente $\alpha$ de Crombach de .93. Los valores del índice KaiserMeyer-Olkin (=.89), así como la prueba de esfericidad de Bartlet con $\chi^{2}=20995.73, g l=171 p<.001$, han indicado la pertinencia de realizar un análisis factorial.

Se han obtenido tres factores que explican el $79.70 \%$ de la varianza total, y cada ítem tiene una carga factorial elevada en uno solo de los factores. En la Tabla 1 se muestra la matriz de factores rotada a la que dan lugar los ítems del cuestionario definitivo, reenumerados tal y como fueron posteriormente aplicados a los estudiantes de GI en la segunda fase del estudio. A partir de la matriz de la Tabla 1, se han nombran e interpretado los tres factores:

Factor 1: Ansiedad ante la evaluación. Se refiere a la ansiedad que experimenta el individuo al ser evaluado (exámenes, ansiedad social, actuación si interviene un compañero, familiar, profesor ....). Incluye los ítems: 3 , $5,6,7,10,11,13,14,15,17,18$.

Factor 2: Ansiedad hacia los números y las operaciones matemáticas. Es la ansiedad al resolver problemas y ejercicios de Matemáticas. Incluye los ítems: 2, 8, 9, 12.

Factor 3: Ansiedad ante la temporalidad. Hace referencia a la ansiedad que siente el alumnado al pensar en el tiempo que falta para hacer un examen o llevar los ejercicios hechos para clase. Incluye los ítems: 1, 4, 16, 19.

Tabla 1

Matriz de factores rotados para $3^{\circ}$ y $4^{\circ}$ de ESO

\begin{tabular}{|c|c|c|c|c|}
\hline \multirow[b]{4}{*}{ Ítems } & \multirow{4}{*}{$\begin{array}{l}\% \text { de varianza explicada: } \\
\text { Coeficiente } \alpha \text { de Cronbach: }\end{array}$} & \multicolumn{3}{|c|}{ Factor } \\
\hline & & 1 & 2 & 3 \\
\hline & & 46.96 & 17.21 & 15.53 \\
\hline & & .98 & .95 & .74 \\
\hline 5 & $\begin{array}{l}\text { Me pongo nervioso cuando alguien me mira mientras hago los deberes de } \\
\text { Matemáticas }\end{array}$ & .982 & & \\
\hline 18 & Me siento nervioso cuando tengo que explicar un problema en clase de Matemáticas & .970 & & \\
\hline 7 & Me ponen nervioso los exámenes de Matemáticas & .963 & & \\
\hline 4 & Me siento nervioso cuando hago un examen de evaluación de Matemáticas & .960 & & \\
\hline 15 & Estoy nervioso al recibir las notas finales (del examen) de Matemáticas & .945 & & \\
\hline 11 & Me pongo nervioso cuando hago el examen final de Matemáticas & .938 & & \\
\hline 10 & Me siento nervioso al tener que explicar un problema de Matemáticas al profesor & .936 & & \\
\hline 17 & $\begin{array}{l}\text { Me siento nervioso cuando nos ponen un problema y un compañero lo acaba antes } \\
\text { que yo }\end{array}$ & .872 & & \\
\hline 13 & $\begin{array}{l}\text { Me siento nervioso cuando intento comprender a otro compañero explicando un } \\
\text { problema de Matemáticas }\end{array}$ & .789 & & \\
\hline 3 & $\begin{array}{l}\text { Me siento nervioso cuando escucho cómo otros compañeros resuelven un problema } \\
\text { de Matemáticas }\end{array}$ & .741 & & \\
\hline 6 & Me pongo nervioso cuando me pongo a estudiar para un examen de Matemáticas & .680 & & \\
\hline 9 & Me pone nervioso hacer operaciones Matemáticas & & .931 & .291 \\
\hline 12 & Me siento nervioso cuando me dan una lista de ejercicios de Matemáticas & & .919 & .305 \\
\hline 8 & $\begin{array}{l}\text { Me siento nervioso cuando me ponen problemas difíciles para hacer en casa y que } \\
\text { tengo que llevar hechos para la siguiente clase }\end{array}$ & .218 & .831 & .351 \\
\hline 2 & $\begin{array}{l}\text { Me pongo nervioso cuando abro el libro de Matemáticas y encuentro una página } \\
\text { llena de problemas }\end{array}$ & & .736 & \\
\hline 4 & $\begin{array}{l}\text { Me pongo nervioso cuando me doy cuenta de que el próximo curso aún tendré clases } \\
\text { de Matemáticas }\end{array}$ & & .268 & .854 \\
\hline 19 & Me siento nervioso cuando empiezo a hacer los deberes & & & .823 \\
\hline 1 & $\begin{array}{l}\text { Me siento nervioso al pensar en el examen de Matemáticas, cuando falta una hora } \\
\text { para hacerlo }\end{array}$ & & .264 & .816 \\
\hline 16 & Me siento nervioso cuando quiero averiguar el cambio en la tienda & & & .734 \\
\hline
\end{tabular}

Nota: Se omiten los valores $<0.2$.

Nótese además la pertinencia de, dada la diferencia en las edades y por consiguiente en la maduración personal, realizar un AFE sobre una muestra diferente a la usada en el trabajo de Muñoz-Cantero y Mato- 
Vázquez (2006) para validar la escala original. En efecto, se pasa de 5 a sólo 3 tres factores. Es más, tal como se puede comprobar en la Tabla 2, la distribución de los ítems no ha coincidido en las dos escalas. Por ejemplo, el factor "Ansiedad hacia los números y las operaciones Matemáticas" no tiene en común ningún ítem en las dos escalas.

\section{Tabla 2}

\section{Comparación entre cuestionarios original y modificado}

\begin{tabular}{lcc}
\hline & Ítems (Original) & Ítems (Modificado) \\
\hline Ansiedad hacia la evaluación & $1,2,8,10,11,14,15,18,20,22,23$ & $3,5,6,7,10,11,14,15,17,18$ \\
Ansiedad ante la temporalidad & $4,6,7,12$ & $1,4,16,19$ \\
$\begin{array}{c}\text { Ansiedad hacia la comprensión de } \\
\text { problemas matemáticos }\end{array}$ & $5,17,19$ & \\
$\begin{array}{c}\text { Ansiedad hacia los números y las } \\
\text { operaciones matemáticas }\end{array}$ & $3,13,16$ & $2,8,9,12$ \\
$\begin{array}{c}\text { Ansiedad hacia situaciones } \\
\text { matemáticas de la vida real }\end{array}$ & $9,21,24$ & \\
\hline
\end{tabular}

\section{Análisis Factorial Confirmatorio}

En primer lugar, se analizó el modelo especificado en la etapa anterior, con 19 variables endógenas observables y tres variables endógenas correspondientes a los factores obtenidos en la etapa anterior. Al testar el supuesto de normalidad multivariante, se obtubo una critical ratio multivariada de 27.55 , lo que superó el valor de referencia de 5.00 sugerido en Bentler (2005) a partir del cual se recomienda no asumir normalidad en los datos. Esto incide severamente en los test basados en varianzas y covarianzas (Decarlo, 1997), haciendo poco fiables las interpretaciones basadas en el método de máxima verosimilitud (Byrne, 2010). Mediante la distancia de Mahalanovis no se detectaron oultiers, pero el tamaño de la muestra de 215 individuos no multiplicaba por 10 los 41 parámetros estimados en el modelo, por lo que no era viable usar el método de Distribución Asintótica Libre (Raykow y Marcoulides, 2006). Asimismo, tampoco se empleó bootstrapping, debido al tamaño de la muestra (Ichikawa y Konishi, 1995).

Se empleó entonces el método de mínimos cuadrados no ponderados (ULS), que no requiere normalidad multivariable, con el que se obtuvo una matriz de covarianzas entre factores con los siguientes autovalores: $.033, .038,0.82$. Teniendo en cuenta los posibles errores debidos a la aplicación de métodos numéricos, se interpretó que la matriz de varianzas-covarianzas es semidefinida positiva. Por lo tanto, según lo expuesto en Blunch (2008), los datos podían explicarse con menos dimensiones. En base a lo expresado por los ítems del Factor 3, se optó por redistribuirlos entre los otros dos factores.

El ítem 1 se refiere a la evaluación, por lo que se incluyó en el Factor 1 "Ansiedad ante la evaluación". Los ítems 16 y 19 se refieren a la realización de actividades Matemáticas, por lo que se incluyó en el Factor 2 “Ansiedad hacia los números y las operaciones Matemáticas" (ver Tabla 2). Por su parte, el ítem 4 "Me pongo nervioso cuando pienso en volver a tener clases de Matemáticas el próximo curso" puede aludir tanto a la actividad matemática académica como a la evaluación que ésta implica. Se compararon entonces dos modelos de dos factores, el Modelo A, donde el ítem 4 se incluía en el Factor "Ansiedad ante la evaluación", y el Modelo B (Figura 1) con (i4) en el Factor "Ansiedad hacia los números y las operaciones Matemáticas".

Repitiendo todos los análisis con el método ULS, todas las matrices de covarianzas resultaron definidas positivas. Ambos modelos estaban sobre-identificados, al tener 39 parámetros para estimar y 151 grados de libertad, convergiendo el método en 4 interacciones. Los pesos de regresión no estaban próximos a 1, por lo que no se infringía la viabilidad de los valores.

Para el estudio del ajuste de los modelos y su comparación se calcularon distintos índices (Tabla 4) cuyos valores fueron interpretados siguiendo lo expuesto en (West, Taylor y Leona, 2012). Se calcularon también los valores para el modelo con todos los ítems en un único factor, al que se llamó Modelo C y se compararon los tres modelos alternativos (Tabla 3). 


\section{Figura 1}

Modelo de ecuaciones estructurales para el Modelo B.

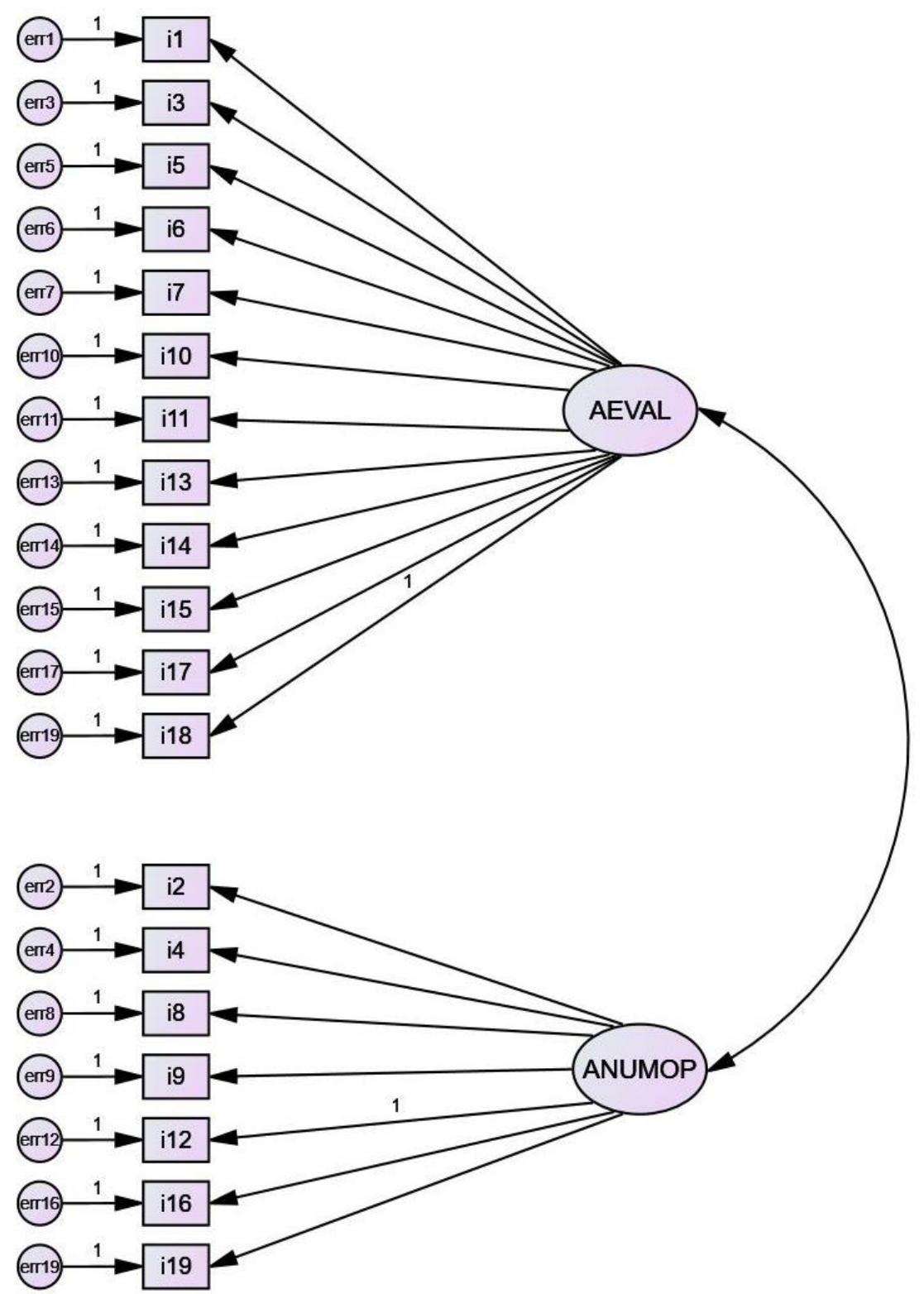

Nota: Se consideran dos variables latentes relacionadas entre sí: $\mathrm{AEVAL}=$ Ansiedad ante la evaluación y ANUMOP = Ansiedad ante los números y las operaciones matemáticas

\section{Tabla 3}

Índices de ajuste para los modelos comparados.

\begin{tabular}{lccccccccc}
\hline Modelo & $\chi^{2}$ & RMR & GFI & AGFI & NFI & RFI & PRATIO & PGFI & PNFI \\
\hline Modelo A & 633.247 & .125 & .956 & .944 & .938 & .930 & .883 & .759 & .828 \\
Modelo B & 616.898 & .123 & .957 & .946 & .939 & .931 & .883 & .760 & .830 \\
Modelo C & 643.911 & .126 & .955 & .944 & .937 & .930 & .938 & .764 & .930 \\
Modelo saturado & .000 & .000 & 1.00 & & 1.00 & & .000 & & .000 \\
Modelo de independencia & 10182.128 & .500 & .287 & .208 & .000 & .000 & 1.00 & .250 & .000 \\
Modelo cero & 14276.260 & & .672 & .000 & & & & .000 & \\
\hline
\end{tabular}


En primer lugar, por ser el Modelo B es el que tuvo asociados valores para $\chi^{2}$ y RMR con menor valor absoluto, fue el modelo que tuvo la matriz teórica de covarianzas asociada más próxima a la matriz de covarianzas observada.

Por otra parte, el Modelo B es el que tuvo valores para los índices GFI y AGFI más próximos a 1, indicando por tanto un mejor ajuste. Nótese la complementariedad de estos índices con el RMR, pues este último no usa matrices de pesos. Fue también el Modelo B el que tuvo valores más altos para los índices incrementales NFI y RFI, resultando el modelo más distante del Modelo Nulo de entre los tres comparados.

Finalmente, si bien el Modelo C tenía asociados los valores más próximos a uno en los índices de ajuste de parsimonia, recordemos que en él todos los ítems se agrupaban en un único factor. Entre los modelos con dos factores, el Modelo B tuvo valores más próximos a uno (PGFI y PNFI) o iguales (PRATIO) que el Modelo A.

En resumen, se ha obtenido que la ansiedad hacia las Matemáticas en estudiantes de GI se estructura en dos factores siguiendo el Modelo B (Figura 1, con correlación entre factores .857).

\section{Tabla 4}

Estadísticos descriptivos Modelo B.

\begin{tabular}{lcccccccc}
\hline & $M$ & $E E M$ & $M o$ & $P$ 25 & Mdna & $P$ 75 & $D T$ & Curtosis \\
\hline Ansiedad Evaluación & 2.44 & .57 & 2.25 & 1.75 & 2.33 & 2.92 & 0.84 & -0.28 \\
Ansiedad Numérica & 1.71 & .05 & 1 & 1.14 & 1.57 & 2.14 & 0.66 & 0.35 \\
\hline
\end{tabular}

En los estadísticos descriptivos para los factores del Modelo B (Tabla 4) se observa que los niveles medios de ansiedad son bajos para la "Ansiedad hacia los números y operaciones", (entre 1 = Nada y 2 = Un Poco). Además, la mediana y el percentil 75 de ese factor indican que gran parte de los/as estudiantes se concentra en esos niveles bajos. Para la "Ansiedad ante la evaluación”, la media y los percentiles son más elevados. Esto implica una distinción entre ambos factores. A la vista de los resultados, se revisó más pormenorizadamente los ítems del factor "Ansiedad ante la evaluación" y se observó que los de mayor media y mediana son aquellos en los que aparece la palabra "examen".

Para estudiar la posible relación lineal entre ambos factores, dado el tamaño de la muestra asumimos distribución normal y obtenemos un coeficiente de correlación de Pearson $r=0.701$ con $p<.001$. Entonces, la relación entre ambos factores existe, pero es ligera.

\section{Discusión}

En este trabajo se ha determinado la estructura factorial de la ansiedad hacia las Matemáticas en estudiantes de Ingeniería. En efecto, esta ha seguido el Modelo B (Figura 1), que consta de dos factores: "Ansiedad ante la evaluación" y "Ansiedad hacia los números y las operaciones Matemáticas". Además, esto permite medir la ansiedad, no sólo en esta muestra, de la que se han expuesto los resultados, sino también en otras poblaciones con características similares, como estudiantes universitarios de aquellas titulaciones donde las asignaturas sobre Matemáticas tengan un cierto peso en el currículum.

El aspecto relativo a las características de la población es relevante. De hecho, los resultados de este artículo han establecido diferencias entre los/as estudiantes de ingeniería y otras poblaciones, como el alumnado de ESO. En primer lugar, en poblaciones de ESO se encontraron niveles más altos (Broc-Cavero, 2006; FierroHernández, 2006; Gómez-Chacón, 2007;). Ahora bien, si se tiene en cuenta que los individuos investigados procedían del Bachillerato Tecnológico, los índices obtenidos no son tan bajos, pues su nivel de agrado hacia la asignatura debería ser más alto. No obstante, se ha de tener presente que encontrar ansiedad hacia los exámenes es frecuente en diferentes etapas educativas (Akey, 2006; Muñoz-Cantero y Mato-Vázquez, 2006).

Ahondando en la diferencia entre poblaciones, en este trabajo se ha puesto de manifiesto que la propia estructura del constructo, la conceptualización de la noción de ansiedad hacia las Matemáticas, es diferente para los/as estudiantes de Ingeniería que para los/as de ESO. Para estos últimos, Muñoz-Cantero y MatoVázquez (2006) obtuvo cinco factores, mientras que con los estudiantes universitarios he han obtenido sólo 
dos. Entre los elementos que han influido en la conceptualización de la ansiedad pudiera figurar el gusto por las Matemáticas o el rendimiento académico (Bazán, 2006), ya que la muestra de ESO estaba formada por alumnos/as muy diversos/as mientras que la mayor parte de los/las estudiantes de Ingeniería procedían del Bachillerato Tecnológico (171 de 215).

Por otra parte, los estadísticos descriptivos calculados para los/las estudiantes de Ingeniería, reforzarían la diferenciación de dos factores. En concreto, los valores de la media, la moda y los percentiles han sido mayores en el factor "Ansiedad ante la evaluación" que para "Ansiedad hacia los números y operaciones". Esto estaría en línea con los resultados de otros estudios en los que se ha obtenido que el alumnado experimenta mayor ansiedad al realizar actividades matemáticas cuando se siente evaluado por otros/as (Murillo-Torrecilla y Hernández-Castilla, 2011).

También se reflejaron diferencias al comparar cada ítem por separado. A través de la media y la mediana ha podido observarse cómo lo expresado por algunos ítems ha sido percibido como fuente de ansiedad, mientras que lo expresado por otros no. En particular, los niveles de ansiedad más altos se han observan en aquellos ítems que aluden a la realización de exámenes finales o a los resultados de estos. Por el contrario, el hecho de ver cómo un/una compañero/a resuelve un problema más rápido no provocaría ansiedad.

La relación lineal entre factores ha resultado leve. Esta conjunción de resultados tendría implicaciones docentes. Consideramos que la relación entre factores debería ser tenida en cuenta, pues un aumento de ansiedad hacia los números y las operaciones podría desencadenar un aumento en la ansiedad ante los exámenes (Kazelskis, 2000). Siguiendo este argumento, cobraría importancia el clima en el aula universitaria, la autoconfianza del alumnado y la adecuación de la carga de trabajo. Estos elementos, al relacionarse con la ansiedad en el momento de la evaluación, indirectamente influirían en los resultados académicos (ImmordinoYang y Damasio, 2007; Swars et al., 2010).

Una correlación baja entre los factores indicaría al profesorado universitario de Matemáticas que la ansiedad de sus estudiantes puede no deberse al contenido específico de la asignatura sino al proceso de evaluación, de ahí los dos factores diferenciados. De hecho, aunque las Matemáticas son poco populares entre el estudiantado universitario (Delgado et al., 2017), en general se considera capacitado para aprenderlas, las valora y su actitud es positiva en las Enseñanzas Técnicas (Pérez-Tyteca et al., 2011).

Aunque serían necesarias más investigaciones, los estadísticos de la Tabla 4 sugieren que el método de evaluación pudiera incidir en la ansiedad. En este sentido, los ítems que contienen la palabra "examen" en su redacción han alcanzado una media y mediana mayores que los ítems que se refieren a procesos de evaluación diferentes. Por ejemplo, las puntuaciones para el ítem "Me pongo nervioso cuando hago el examen final de Matemáticas" alcanzaron una media de 3.25 y mediana de 2.5. Por el contrario, "Me siento nervioso al tener que explicar un problema de Matemáticas al profesor" presentó de media 2.65 y mediana 2 y "Me siento nervioso cuando tengo que explicar un problema en clase de Matemáticas" media 2.5 y mediana 2. Esto coincide con otros resultados, que indican que una evaluación continua provoca menos ansiedad que la evaluación mediante exámenes (Yara, 2009).

Por ello, cabe sugerir al profesorado de Matemáticas en una titulación de Ingeniería que contemple, en la medida de lo posible, la posibilidad de usar algún proceso de evaluación que relativice la trascendencia del examen final. En este sentido, el cuestionario que se ha presentado en este estudio, de fácil aplicación en el aula, permitiría al profesorado conocer los niveles de ansiedad hacia las matemáticas de sus estudiantes en concreto y tener así un elemento de decisión a la hora de optar por una u otra metodología.

Limitaciones de la investigación. En la segunda fase, el presente estudio ha sido realizado con estudiantes de un único grado en Ingeniería y de una única universidad. lo que podría suponer una merma en la representatividad de la muestra.

Nuevas líneas de investigación. A partir del presente trabajo ser podrían realizar en el futuro estudios para comparar los resultados obtenidos en función de características específicas de cada muestra. Por ejemplo, se podrían considerar muestras de estudiantes de grados de Ingeniería distintos, y ver luego si existen diferencias en la conceptualización de la Ansiedad hacia las Matemáticas y los niveles alcanzados en función del grado 
concreto. Del mismo modo, sería de interés realizar estudios análogos en varias universidades, tanto públicas como privadas y de distintas zonas geográficas, para indagar posteriormente si existen diferencias dependiendo de estas variables.

\section{Referencias}

Akey, T. (2006). School context, student attitudes and behavior, and academic achievement: An exploratory analysis. New York: MDRC. Disponible en: https://www.mdrc.org/publication/student-context-studentattitudes-and-behavior-and-academic-achievement

Álvarez, Y., \& Ruíz, M. (2010). Actitudes hacia las matemáticas en estudiantes de ingeniería en universidades autónomas venezolanas. Revista de Pedagogía, 31, 225-249. http://ve.scielo.org/scielo.php?script=sci_art text\&pid=S0798-97922010000200002

Anderson, J. R., \& Schunn, C. (2000). Implications of the ACT-R learning theory: No magic bullets. En R. Glaser (Ed.), Advances in instructional psychology. Educational design and cognitive science (Vol. 5), (pp. 1-33). Mahwah, NJ: Erlbaum. Recuperado de: Christian Schumn: Publications, University of Pittsburg: http://www.lrdc.pitt.edu/schunn/papers/NoMagicBullets.pdf

Ashcraft, M. (2002). Math Anxiety: Personal Educational and Cognitive Consequences. Current directions in psychological science, 11(5), 181-185. https://doi.org/10.1111/1467-8721.00196

Auzmendi, E. (1992). Las actitudes hacia la matemática-estadística en las enseñanzas medias y universitarias. Características y medición. Bilbao: Mensajero.

Bazán, J. L. (2006). Las actitudes hacia la Matemática-Estadística dentro de un modelo de aprendizaje. Revista Semestral del Departamento de Educación, 25(28), 7-20. http://revistas.pucp.edu.pe/index.php/educacion/ article/view/2041

Bentler, P. (2005). EQS 6 Structural equations program manual. Encino, CA: Multivariate Software. Disponible en: http://www.mvsoft.com/pub/EQS61QuickStart.pdf

Birgin, O., Çatlioglu, H., Costu, S., \& Aydin, S. (2009). The investigation of the views of student mathematics teachers towards computer-assisted mathematics instruction. Procedia Social and Behavioral Sciences, 1 , 676-680. https://doi.org/10.1016/j.sbspro.2009.01.118

Blunch, N. (2008). Introduction to Structural Equation Modelling using SPSS and AMOS. London: SAGE Publications.

Broc-Cavero, M. Á. (2006). Motivación y rendimiento académico en alumnos de Educación Secundaria Obligatoria y Bachillerato LOGSE. Revista de Educación, 340. 379-414. http://www.educacionyfp.gob.es/ revista-de-educacion/numeros-revista-educacion/numeros-anteriores/2006/re340/re340-13.html

Byrne, B. (2010). Structural Equation Modeling with AMOS. Basic concepts, Applications, and Programming. (2 Edition). New York: Routledge.

Carmona, J. (2004). Una revisión de las evidencias de fiabilidad y validez de los cuestionarios de actitudes hacia la estadística. Statstics Education Research Journal. 3(1), 5-28. http://iase-web.org/documents/SERJ/ SERJ3(1)_marquez.pdf?1402525004

Clark, D., \& Beck, A. (2012). Terapia cognitiva para trastornos de ansiedad. Bilbao: Desclée de Brouwer.

Danhke, G., L. (1989). Investigación y comunicación. En C. Fernández-Collado, \& G.L. Danhkle (Eds.). La comunicación humana: ciencia social (385-454). México, D.F.: McGraw-Hill de México.

Decarlo, L. (1997). On the meaning and use of kurtosis. Psychological Methods, 2, 292- 307. https://doi.org/ 10.1037/1082-989X.2.3.292

Delgado, I., Espinoza, J., \& Fonseca, J. (2017). Ansiedad matemática en estudiantes universitarios de Costa Rica y su relación con el rendimiento académico y variables sociodemográficas. Propósitos y Representaciones, 5(1), 275-324. https://doi.org/10.20511/pyr2017.v5n1.148

Eccius-Wellmann, C., \& Lara-Barragán, A. (2016). Hacia un perfil de ansiedad matemática en estudiantes de nivel superior, Revista Iberoamericana de Educación Superior, 7, 109-129. https://doi.org/10.22201/iisue. 20072872e.2016.18.179 
Fennema, E., \& Sherman, J. (1976). Mathematics Attitudes Scales: Instruments Designed to Measure Attitudes toward the Learning of Mathematics by Females and Males. Journal for Research in Mathematics Education, 7(5), 324-326 https://doi.org/10.2307/748467

Fierro-Hernández, C. (2006). Valoración del impacto de un programa de educación en valores en el último curso de Educación Secundaria Obligatoria. Revista de Educación, 339, 455-466. http://www.educacionyfp. gob.es/revista-de-educacion/numeros-revista-educacion/numeros-anteriores/2006/re339/re339-19.html

Gómez-Chacón, I. M. (2007). Sistema de creencias sobre las Matemáticas en alumnos de Secundaria. Revista Complutense de Educación, 18, 125-143. https://revistas.ucm.es/index.php/RCED/article/view/RCED0707 220125A

Hancock, D. (2001). Effects of test anxiety and evaluative threat on students' achievement and motivation. Journal of Educational Research, 94(5), 284-290. https://doi.org/10.1080/00220670109598764

Ichikawa, M., \& Konishi, S. (1995). Application of the Bootstrap Methods in Factor Analysis. Psychometrika, 60, 77-93. https://doi.org/10.1007/BF02294430

Immordino-Yang, M. H., \& Damasio, A. (2007). We Feel, Therefore We Learn: The Relevance of Affective and Social Neuroscience to Education. Mind, Brain, and Education, 1(1), 3-10. https://doi.org/10.1111/ j.1751-228X.2007.00004.X

Iossi, L. (2007). Strategies for reducing math anxiety in post-secondary students. En S. M. Nielsen, \& M. S. Plakhotnik (Ed.), Proceedings of the sixth annual College of Education Research Conference: Urban and International Education Section (pp. 30-35). Miami: Florida International University, 30-35. Disponible en: https://pdfs.semanticscholar.org/56e1/e2faf9d8511fc10052a780e26b0dafc4ead2.pdf

Kazelskis, R. (2000). Mathematics anxiety and test anxiety: Separate constructs? Journal of Experimental Education, 68 (2), 137-146. https://doi.org/10.1080/00220970009598499

Muñoz-Cantero, J. M., \& Mato-Vázquez, M. D., (2006). Diseño y validación en un cuestionario para medir las actitudes hacia las matemáticas en alumnos de ESO, Revista Galego-Portuguesa de Psicoloxía e Educación, 13, 413-424. http://hdl.handle.net/2183/7048

Murillo-Torrecilla, F. J., \& Hernández-Castilla, R. (2011). Efectos escolares de factores socio-afectivos. Un estudio multinivel para Iberoamérica. Revista de Investigación Educativa, 29(2), 407-427. https://revistas.um.es/rie/article/view/111811

National Council of Teachers of Mathematics (NCTM, 2003). Principios y Estándares para la educación matemática. Sevilla: S.A.E.M. "Thales".

Nortes, R., \& Nortes, A. (2014). Ansiedad hacia las Matemáticas, agrado y utilidad en futuros maestros. En M. T. González, M. Codes, D. Arnau, \& T. Ortega (Eds.), Investigación en Educación Matemática XVIII (pp. 485-492). Salamanca: SEIEM. Disponible en: http://funes.uniandes.edu.co/6089/1/Nortes2014 AnsiedadSEIEM.pdf

Pérez-Tyteca, P., Castro, E., Rico, L., \& Castro, E. (2011). Ansiedad matemática, género y ramas de conocimiento en alumnos universitarios. Enseñanza de las ciencias, 29(2), 237-250. https://doi.org/ $10.5565 / \mathrm{rev} / \mathrm{ec} / \mathrm{v} 29 \mathrm{n} 2.570$

Raykow, T., \& Marcoulides, G. (2006). A first course in structural equation modeling (2nd ed.). Mahwah, N.J.: Lawrence Erlbaum Associates.

Richardson, F., \& Suinn, R. (1972). The Mathematics Anxiety Rating Scale. Journal of Counseling Psychology, 19, 551-554. https://doi.org/10.1037/h0033456

Swars, S. L., Daane, C. J., \& Giesen, J. (2010). Mathematics anxiety and mathematics teacher efficacy: What is the relationship in elementary preservice teachers? School Science and Mathematics, 106(7), 306-315. https://doi.org/10.1111/j.1949-8594.2006.tb17921.x

Tobias, S. (1985). Test anxiety: Interference, defective skills, and cognitive capacity. Educational Psychologist, 20, 135-142. https://doi.org/10.1207/s15326985ep2003_3

Van Eerde, W. (2003). A meta-analytically derived nomological network of procrastination. Personality and Individual Differences, 35, 1401-1418. https://doi.org/10.1016/S0191-8869(02)00358-6 
West, S., Taylor, A., \& Leona, W. (2012). Model fit and model selection in structural equation modeling. En R. H. Hoyle, (Ed.), Handbook of Structural Equation Modeling (pp. 209-231). New York: The Guilford Press.

Yara, P. O. (2009), Mathematics anxiety and academic achievement in some selected senior secondary schools in Southwestern Nigeria. Pakistan Journal of Social in Sciences, 6, 133-137. https://medwelljournals.com/ abstract/?doi=pjssci.2009.133.137

Fecha de recepción: 31 de marzo de 2020.

Fecha de revisión: 8 de mayo de 2020.

Fecha de aceptación: 16 de mayo de 2020.

Fecha de publicación: 1 de julio de 2020. 